\title{
Verfassungsrechtliche Perspektiven des dualen Rundfunksystems
}

\author{
Helge Rossen-Stadtfeld
}

Der Wandel der Strukturen gesellschaftlicher Kommunikation beeinflusst auch die Entwicklungsperspektiven des deutschen dualen Rundfunksystems. Hierauf reagieren zwei verfassungsrechtlich begründete Vorschläge zur Neuordnung des Rundfunksystems. Der eine Vorschlag zielt auf die Kombination eines deregulierten kommerziellen Marktrundfunks mit einem öffentlich-rechtlichen Kulturrundfunk. Nach der anderen Konzeption soll grundsätzlich auf rundfunkspezifische Regulierung verzichtet, zugleich aber kulturelle Vielfalt gezielt gefördert werden. Den normativen Vorgaben, die in der Rechtsprechung des Bundesverfassungsgerichts zu Art. 5 Abs. 1 S. 2 GG entwickelt worden sind, genügen beide Ansätze nicht; sie können das Modell, das diesen Vorgaben zugrunde liegt, nicht ersetzen.

Keywords: Rundfunkrecht, duales System, Rundfunksystem, Konvergenz, Marktrundfunk, Kulturrundfunk

Die Entwicklungsperspektiven des deutschen dualen Rundfunksystems erscheinen derzeit besonders unsicher. Die Umwelt dieses Systems ist durch vermachtete Weltmärkte, global operierende Medienkonzerne, Kommerzialisierungstendenzen, starken - technisch, wirtschaftlich und politisch induzierten - Konvergenzdruck und nicht zuletzt auch den Zusammenbruch der „new economy“ geprägt. Sie verändert sich schnell und tief greifend. Das alles ist auch von der Diskussion um verfassungsrechtliche Grundlagen, Spielräume und Ziele einer Ausgestaltung des deutschen Rundfunksystems zur Kenntnis genommen worden. Daraufhin zeichnet sich eine neue Entwicklungsstufe des dualen Modells ab: ein marktnah eingerichteter Unterhaltungsrundfunk, der eine mehr oder weniger ausgeweitete Kulturnische aufweist (Bullinger, 2001; Vesting, 2001a).

\section{Fortschritt zum Marktmodell?}

Ausgangspunkt dieser neueren Ansätze ist die Mahnung, die Wirklichkeit einer „Ökonomie der Aufmerksamkeit“ (Franck, 1998) - geprägt durch oligopolisierte Produktionsstrukturen und die Ausrichtung auf möglichst billige und quotenstarke Unterhaltungsprogramme - zu akzeptieren, realitätsferne duale Konstruktionen fallen zu lassen und auf realitätsnähere Modelle umzustellen. So rückt das Marktmodell wieder in den Vordergrund der rundfunkrechtlichen Auseinandersetzung.

\section{Marktrundfunk als „Normalfall“?}

Die Möglichkeit hierzu eröffnet zum einen die grundrechtstheoretische Prämisse, es seien die Grundrechte in erster Linie reine Abwehrrechte, gerichtet gegen einen Staat, der durch sie grundsätzlich an jedem Eingriff in bürgerliche Freiheiten gehindert werde. Da die Knappheitsprobleme heute nicht mehr bestünden, die ein gesetzlich befestigtes öffentlich-rechtliches Rundfunkmonopol zunächst hätten rechtfertigen können, verlange Art. 5 Abs. 1 Satz 2 GG nach einem Abbau aller normativen Hemmnisse, die einer nur übergangsweise und vorläufig dispensierten Rundfunkunternehmerfreiheit bislang ge- 
zogen worden seien. Das andere Argument, mit dem das Marktmodell als Gestaltungsvorgabe plausibilisiert werden soll, macht ein allmähliches Zusammenwachsen der europäischen Rundfunkordnungen geltend. In diesem Vorgang erscheint der Rundfunk im Kern: die im Programm eingefangene Aufmerksamkeit möglicher Kunden derjenigen Unternehmen, die Werbezeit eingekauft haben (Heinrich, 1999, S. 277 ff.) - als ein Wirtschaftsgut unter anderen in einer europäischen Wirtschaftsordnung und die nationale Rundfunkordnung als Teil eines überwölbenden Rundfunk-Binnenmarktes (dazu Hoffmann-Riem, 2000, S. 74 ff. m. w. N.). Zusammengenommen erlauben das grundrechtstheoretische und das europarechtliche Argument die Anmahnung der „Normalität" eines nach dem Marktmodell ausgestalteten Rundfunkwesens (so bei Schoch, 1998, 193 ff.; s. auch Starck, 1999, Rn. 115 ff.; in der Grundtendenz auch Bullinger/Mestmäcker, 1996, S. 71 ff.). Das duale Rundfunksystem offenbart sich unversehens wieder als rechtfertigungsbedürftiger Sonderfall, womöglich als Abirrung. Korrekturen in die Richtung auf den marktnäheren Normalfall erscheinen dann nahe liegend und selbstverständlich.

Dieses Ergebnis ist nicht neu (so zu Recht Eifert, 2002, S. 60). Die Behauptung einer verkannten und daraufhin wieder in ihr Recht zu setzenden marktwirtschaftlich-wettbewerblichen „Normalität“ begleitet die Entwicklung des dualen Rundfunksystems seit dessen Beginn, wenn auch zum Teil auf weitere oder anders gewichtete Argumente gestützt. Auch für die jüngeren Ausprägungen, in denen diese ältere Behauptung heute erhoben wird, gelten deshalb die Einwände weiter, die immer schon gegen die Forderung eines reinen Marktrundfunks vorgebracht worden sind (Diskussion bei HoffmannRiem, 2000, S. 116 ff., 171 ff. m. w. N.).

\section{Verzicht auf rundfunkspezifische Vermittlung?}

Die Einwände gegen eine rundfunkverfassungsrechtliche Normalität nach Maßgabe des Marktmodells leiten sich vor allem aus der Feststellung ab, dass in einem System ökonomischen Wettbewerbs letztlich die Funktion nicht erfüllt würde, die Art. 5 Abs. 1 GG in seiner Auslegung durch das Bundesverfassungsgericht dem Rundfunk zuweist. In einem nach dem Marktmodell ausgestalteten System ist das Programmangebot in erster Linie darauf ausgerichtet, das Versprechen einer möglichst weit reichenden und stabilen Aufmerksamkeitsbindung so überzeugend erscheinen zu lassen, dass stets in hinreichendem Umfang Werbegelder eingeworben werden können. In den verbleibenden bislang allerdings sehr kleinen - Teilbereichen der Entgeltfinanzierung wird das Programm selbst unmittelbar zur Ware, die auf möglichst gute Marktgängigkeit bei niedrigen Erstellungskosten hin produziert und angeboten werden muss. In beiden Fällen ist der Rundfunk nicht mehr Medium und Faktor freier, chancengleicher und umfassender Meinungsbildung, ob in öffentlichen oder in individuell-privaten Bezügen. Das Marktmodell und das ihm zugrunde liegende Paradigma wirtschaftlichen Wettbewerbs erlauben von vornherein schon gar keine Aussagen zur Erfüllbarkeit der Funktion, die dem Rundfunk im Hinblick auf die kommunikationsverfassungsrechtliche Basisgewährleistung der Meinungsbildungsfreiheit normativ zugeschrieben wird. Für die ökonomische Analyse ist allein maßgeblich, dass das Rundfunkprogramm den Konsumentenpräferenzen gemäß produziert und angeboten wird und dass es im Übrigen möglichst geringe Kosten verursacht (Heinrich, 1999, S. 46). Für diese Analyse müssen demgegenüber normativ aufgeladene Ziele unberücksichtigt bleiben, die sich auf die Ermöglichung freier, umfassender und chancengleicher Meinungsbildung, auf die Gewährleistung vielfältiger Information, Bildung und Unterhaltung sowie ein stetig hohes Qualitätsniveau der Rundfunkprogramme richten. In der Rechtsprechung des Bundesverfassungsgerichts 
gelten diese Zielvorgaben für alle Rundfunkprogramme, auch die kommerziellen Angebote sind nicht von ihnen ausgenommen (BVerfGE 73, 118 [158, 180, 199]; 83, 238 [297, 315 ff.]; 87, 181 [198]).

Die Ausblendung dieser normativen Vorgaben bedarf der Rechtfertigung. Die Option für eine marktnahe „Normalisierung“ des Rundfunkwesens trägt die Beweislast dafür, dass kommunikativ hochgradig ausdifferenzierte Gesellschaften keiner massenmedialen Vermittlungsfunktion mehr bedürfen. Es muss gezeigt werden, dass eine Vermittlungsfunktion jedenfalls mit dem anspruchsvollen Gehalt obsolet geworden ist, den das Bundesverfassungsgericht - in Orientierung an einem Prozessmodell gesellschaftlicher Kommunikation, in dem diese als Zusammenhang selbstregulativer, in fortwährender Umstrukturierung befindlicher gesellschaftlicher Kommunikationsnetzwerke vorgestellt wird - dieser Funktion zugewiesen und als ein Kernelement des Normgehalts von Art. 5 Abs. 1 Satz 2 GG entfaltet hat (seit BVerfGE 12, 205 [260] st. Rspr., s. etwa E 57, 295 [319 ff.]; 83, 238 [295 ff.]; 90, 60 [87 ff.]). Dieser Beweis ist bislang nicht nur nicht erbracht worden. Es gibt vielmehr im Gegenteil zahlreiche Anhaltspunkte dafür, dass die Erschließungs-, Analyse-, Synthetisierungs-, Ergänzungs-, Entdeckungs-, Orientierungs- und mit alledem letztlich Integrationsleistungen, die zur Verwirklichung der Vermittlungsfunktion des Rundfunks erbracht werden müssen, im Zuge der weiteren medientechnischen und medienstrukturellen Entwicklung immer wichtiger werden (Trute, 1998, S. 230 ff., 249 ff.; Holznagel, 1999, S. 39 ff., 118 ff.; Hoffmann-Riem, 2000, S. 136 ff., 311 f.; a. A. Determann, 1999, S. 395 ff.).

\section{Modifikationen des dualen Systems}

Das normative Leitmodell des dualen Rundfunksystems zielt auf lebhafte und vielfaltsfördernde publizistische Konkurrenz (BVerfGE 74, 297 [326]; Diskussion bei Kiefer, 1994). Die tatsächliche Befindlichkeit dieses Systems ist durch starke Tendenzen einer programmlich-inhaltlichen „Konvergenz nach unten“ gekennzeichnet. Der Abstand zwischen Modell und Realisierung ist in der rundfunkrechtlichen Diskussion bemerkt worden. Das hat bislang, soweit erkennbar, zwei Vorschläge einer modelltheoretischen Neuorientierung veranlasst.

\section{Die Zuspitzung: öffentlich-rechtliche Kultur, deregulierte Privatwirtschaft}

Die erste dieser beiden Konzeptionen (Bullinger, 2001) knüpft bei dem Dualismus an, der die derzeitige Ausgestaltung des Rundfunkwesens in Deutschland kennzeichnet, und sucht ihn zuzuspitzen. Ihren Ausgang nimmt diese Konzeption bei einer Schilderung des Wandels im Realbereich der Grundrechtsnormen aus Art. 5 Abs. 1 GG, die für das deutsche duale Rundfunksystem maßgeblich geworden sind. Es wird hingewiesen auf neue Übertragungstechniken bzw. -formen (Digitalisierung) und Übertragungswege (Direktfunksatelliten, Internet), auf die vertikale und horizontale Verflechtung und Konzentration im Bereich der Veranstaltung kommerziellen Rundfunks, auf Anpassungszwänge und Konvergenzdruck im öffentlich-rechtlichen Sektor des dualen Systems, auf ein weltweit verbreitetes, aber individuell abrufbares Angebot zunehmend spezialisierter elektronischer Dienste und schließlich auf die „globale Ökonomisierung der Telekommunikation“ (Bullinger, 2001, S. 198 ff., 200 ff.; ders., 1980; s. auch Vesting, 1997, S. 159 ff., 182 ff., und Reimers, 1999).

Dieser Ausgangsbefund steht nicht mehr im Mittelpunkt medienwissenschaftlicher, medienpolitischer oder medienrechtlicher Diskussionen. Wichtigster Gegenstand sol- 
cher Debatten sind mittlerweile vielmehr die Folgerungen, die aus diesem Befund gezogen werden können oder gar müssen. Auch die hier vorgestellte Konzeption zur Entwicklungsperspektive des dualen Rundfunksystems beruht auf solchen Folgerungen. Sie erscheinen erläuterungsbedürftig.

\section{a) Unklarbeiten und offene Fragen}

Das betrifft schon die Feststellung, es verliere die Knappheit terrestrischer Frequenzen wegen neuer Übertragungswege und -techniken zusehends an Bedeutung (Bullinger, 2001, S. 198). Der beträchtliche Modernisierungsbedarf in den Kabelnetzen und die Unsicherheit bezüglich der weiteren Netzentwicklung (Woldt, 2002; Lauff, 2002) stehen dieser These entgegen. Vor allem aber erschließt sich ihre Relevanz heute nicht mehr von selbst. Immer wieder ist in der Literatur im Anschluss an das FRAG-Urteil (BVerfGE 57, 295 [322]) darauf hingewiesen worden, dass die Rechtfertigung „positiver“ Rundfunkregulierung gerade nicht von dem Fortbestand der Knappheitslagen bei den Übertragungsmöglichkeiten abhänge (s. nur Hoffmann-Riem, 2000, S. 89 ff. m. w. N.). Da sie auf diese Hinweise nicht eingeht, gelangt die Konzeption schon hier in eine Schieflage. Sie meldet weiterhin Regelungsbedarf an, vor allem zur Sicherung eines öffentlich-rechtlichen „nationalen Kulturrundfunks“, aber auch zur Konzentrationskontrolle und zum Jugendschutz im kommerziellen Sektor (Bullinger, 2001, S. 206 ff., 211 ff.). Zugleich aber unterstellt sie dabei unausgesprochen, dass mit der Vermehrung von Übertragungsmöglichkeiten ein deregulierter „,Markt‘ für Rundfunk“ (a.a. O., S. 198) zum Normalfall werde, demgegenüber sich die bestehende Ausgestaltung des dualen Rundfunksystems als normalisierungsbedürftig erweist.

An die Stelle des herkömmlichen „Fernsehvolks“ trete nun eine „im Ansatz spontan agierende und reagierende, ja ,chaotische globale Informationsgesellschaft“, der auch das Prädikat „interaktiv“ zugeschrieben wird (a. a. O. S. 201). Dieser Befund ist so vertraut (Schulz/Held, 2001, S. 111 m. w. N.) wie klärungsbedürftig. In der nationalen Binnenperspektive wird hier der Teil der Bevölkerung als konzeptionell unerheblich erachtet, der sich in der Rolle einer vor dem Fernsehgerät abgelegten „coach potatoe“ wohlfühlt und Interaktionsaufforderungen dann als Zumutung zu empfinden scheint (Dörr/Janik/Zorn, 2002, S. 65 f. m. w. N.). Dabei handelt es sich nach den derzeit verfügbaren Mediennutzungsdaten um die Mehrheit der Gesamtbevölkerung (Ridder/Engel, 2001, S. 107 ff.). Wieder scheint sich die These zu bewahrheiten, dass neue Medien die alten Medien nicht revolutionär ablösen, sondern allenfalls evolutionär ergänzen (Lerg, 1981, in Fortführung einer Spekulation von McLuhan; s. auch Stipp, 1996, S. 5 f.). In einer nach außen gewandten Blickrichtung bleiben ferner die rund zwei Drittel der Weltbevölkerung und ganze Erdteile (Afrika, große Teile Asiens) unberücksichtigt, die nach wie vor keinen gesicherten Zugang zu globalen Netzen haben und diesen, soweit derzeit absehbar, wohl auch nicht erhalten werden (MetzeMangold, 2001).

Problematisch ist der Begriff der „Informationsgesellschaft“ auch aus anderen Gründen. Information entsteht erst im Zusammenhang von Mitteilung und Verstehen, sie ist der bestimmte Sinn, der einem Datum erst noch gegeben werden muss (Steinmüller, 1993, S. 189 ff., 211 ff.). In den modernen Massenmedien und im Internet sind also zunächst keineswegs Informationen, sondern bloße Daten vorfindlich. Letztere müssen erst noch zu Informationen werden. Dieser Prozess ist komplex und voraussetzungsvoll (Hofstadter, 1985, S. 174 ff.; Luhmann, 1984, S. 193 ff.; rechtswissenschaftliche Folgerungen bei Albers, 1996, insbes. S. 117, 121; Schulz, 1998, S. 24 f.). Das wird im Begriff 
der „Informationsgesellschaft“ tendenziell unterschlagen. Gleiches gilt für den Umstand, dass die Befähigung in modernen Gesellschaften erst erworben und dann trainiert werden muss, in den wachsenden Wüsten des Nichtwissens gezielt die Oasen verfügbarer Daten aufzufinden und zu Informationen zu verarbeiten (Rossen-Stadtfeld, 1999, S. 224). Was schließlich ist von dem Begriff der „Informationsgesellschaft“ zu halten, wenn in den damit bezeichneten Gesellschaften die Kluft immer größer wird zwischen denen, die neue Medien und Datenquellen produktiv für sich nützen können, und denen, die das nicht können? Manches spricht dafür, dass es genau diese sich immer weiter öffnende Schere ist, die eine so genannte moderne „Informationsgesellschaft“ kennzeichnet (Kuhlen, 1995; Rötzer, 1999). Alles dies sind Fragen, die auch und gerade in einer rundfunkrechtlichen Perspektive Bedeutung erlangen müssten, die das Leitprinzip freier, umfassender und chancengleicher Meinungsbildung in öffentlichen und privaten Bezügen nicht preisgeben will. Die ungenaue, Ökonomisierungs- und sonstige Vermachtungserscheinungen ausblendende (Michalski, 1997) Rede von der „Informationsgesellschaft“ führt demgegenüber in der Regel dazu, dass diese Fragen von Vornherein ungestellt bleiben.

Schließlich bedürfte auch die „Interaktivität“ genauerer Betrachtung, die hier, wie überhaupt in vielen Darstellungen zum Internet, hervorgehoben wird. Es erschließt sich nicht unmittelbar, in welchem Sinn die Auswahl und Bestellung aus einem Warenkatalog oder die Einholung von Service-, Markt- und Ratgeberinformationen als Interaktion anzusehen sein könnten. In diesen Vorgängen erschöpft sich ein Großteil der auf das Internet bezogenen Nutzungsinteressen (Oemichen, 2002, S. 30 ff.). Jedenfalls erschiene, wenn die hier gemeinte „Interaktivität“ typischerweise in solchen Vorgängen zum Ausdruck käme, der Begriff einigermaßen trivial. Er wäre dann ungeeignet, kühne Visionen einer netzgestützten gesellschaftlichen Kommunikation zu fundieren, und könnte auch nicht den emphatischen Überschuss mit sich führen, auf den die Pragmatik seiner Verwendung doch so häufig setzt. Auch was eine „interaktive globale Gesellschaft“ sein könnte, bleibt undeutlich. Vorderhand gilt immer noch, dass „die Armen schauen, die Reichen senden“ (Krönig, 2001, S. 3; Reljič, 2001) und dass diese „Armen“ - es handelt sich um Rezipienten in den bevölkerungsreichsten Regionen dieser Welt - kaum eine Chance haben, das mediale Angebot einer westlichen Populär- und Kommerzkultur folgenreich „interaktiv“ zu beeinflussen.

\section{b) Zwei eigenständige Rundfunkordnungen}

Es mag sein, dass die Unklarheiten ausgeräumt werden können, die der hier vorgestellten Rundfunkkonzeption noch anhaften. Der Diskussion bedarf aber auch dann noch die Hauptthese und wichtigste Entwicklungsmaßgabe für das duale Rundfunksystem Deutschlands, in der diese Konzeption ausmündet: In der bezeichneten Gesamtentwicklung bedürfe es einer schärferen funktionellen und finanziellen Abgrenzung der beiden Teilbereiche des dualen Rundfunksystems. Auf der einen Seite müsse „im machtvollen Strom globaler Ökonomisierung der Telekommunikation “ ein wirtschaftsferner nationaler und regionaler Rundfunk erhalten bleiben, „als Kristallisationsschwerpunkt für heimische Politik und Kultur und damit für eine nationale, Gesellschaft ““ (Bullinger, 2001, S. 205). Ein aus Gebühren oder vergleichbaren öffentlichen Abgaben finanzierter „nationaler Kulturrundfunk“ sei deshalb einer gesteigerten öffentlich-rechtlichen Pflichtenbindung, gerade auch in programmlicher Hinsicht, zu unterwerfen. Er habe weiterhin „die Gesamtheit der Meinungen und Interessen im Wesentlichen vollständig und ausgewogen zum Ausdruck zu bringen (Ausgewogenheitspflege)“ (a. a. O., S. 211) 
und müsse insoweit „Vorbildwirkung“ auch und gerade den kommerziellen Anbietern gegenüber entfalten können (a. a. O., S. 213 f.; das Petitum einer genaueren Vorgabe des öffentlich-rechtlichen Programmauftrags schon bei Bullinger, 1999, S. 23 f., 43 ff., 115 ff.). Ihm gegenüber sei ein „weltmarktmäßig agierender, aus Werbung und anderen kommerziellen Einnahmen finanzierter privatwirtschaftlicher Rundfunk “ zuzulassen, dessen Pflichtenbindung auf das Maß zurückzuführen sei, „das sich auch im weltweiten Wettbewerb als Sozialbindung rechtfertigen läßt.“ (a. a. O., S. 210). Für dieses reduzierte Pflichtenmaß könne die periodische Massen-Presse als Vorbild dienen (Verpflichtung zu journalistischer Sorgfalt bei Nachrichten und Kommentaren, Jugendschutz), sonstige „marktfremde“ Bindungen müssten freilich aus öffentlichen Mitteln abgegolten werden (a. a. O., S. 212). Zeitliche Werbebeschränkungen seien aufzuheben, da sie ihren Rechtfertigungsgrund in der vielfaltssichernden Aufgabe gefunden hätten, an die der kommerzielle Rundfunk künftig nicht mehr gebunden sei (a. a. O., S. 211 f.). Insgesamt sei dies der Weg, auf dem „die Rundfunkfreiheit von einer nationalen Dienstpflicht zum tragenden Element einer weltoffenen Informationsgesellschaft werden kann“( a. a. O., S. 193).

Ein weitgehend deregulierter kommerzieller Rundfunk wird hier als Fortschritt aus überholten Bindungen, als Normalität der in Art. 5 Abs. 1 S. 2 GG geschützten Freiheit und als letztlich unumgänglich empfohlen. Dieser Rundfunk kann und soll keine „dienende" Funktion mehr im Hinblick auf die Meinungsbildungsfreiheit erfüllen (a. a. O., 2001, S. 215, 217). Er wird in die Freiheit ausschließlich kommerzieller Zielsetzung entlassen. Damit aber wird sich das duale Rundfunksystem den Bedingungen einer „Ökonomie der Aufmerksamkeit" weiter unterwerfen, ohne dass diese Entwicklung durch rundfunkspezifische Sicherungen noch verlangsamt werden könnte. Dass von einem öffentlich-rechtlichen Angebot allein schon deshalb „Vorbildwirkung“ für den kommerziellen Rundfunk ausgehen könnte, weil es qualitativ höherwertig erscheint, ist unrealistisch. Mit der Wirklichkeit eines kommerziellen Rundfunks auf engen und vermachteten Märkten hat diese Überlegung nichts zu tun. Wohl könnte eine Vorbildfunktion des öffentlich-rechtlichen Programmangebots für die externe Beobachtung und Ausgestaltung des Mediensystems nutzbar gemacht werden (Holznagel, 1999, S. 42 f.; Eifert, 2002, S. 71). Ohne eine derart regulative Umsetzung wird sie die funktionale Eingliederung des kommerziellen Rundfunks in die Wirtschaft der Gesellschaft nicht aufbrechen können: Zum Vorbild wird ein öffentlich-rechtliches Angebot erst dann, wenn und soweit dies eine Erhöhung der Quoten erwarten lässt. An letztlich denselben Problemen läuft auch der Vorschlag auf, kommerzielle Veranstalter durch Subventionen zur Produktion und Veranstaltung „marktfremder kultureller Sonderleistungen“ zu veranlassen (Bullinger, 2001, S. 214; Vesting, 2001a, S. 303, s. schon Engel, 1996, S. 63 f., 113). Dieser Vorschlag erscheint zunächst inkonsequent. Das Anliegen der hinter ihm stehenden Konzeption geht gerade dahin, den kommerziellen Rundfunk endlich ganz in eine so weit wie möglich deregulierte Wirtschaftsfreiheit jenseits aller extern vorgegebenen Qualitätsmaßgaben zu entlassen. Vor allem aber ist der Vorschlag bereits praktisch erprobt und hat sich dann als wenig erfolgreich erwiesen (Holznagel, 2002, S. 2355 m. w. N.). Wie sich gezeigt hat, kann ein Rundfunk, sind dessen funktionale Bindungen in der Public Service-Tradition erst einmal gelöst worden, über Subventionen offenbar nicht in vergleichbarer Weise auf ein bestimmtes Programmniveau hingesteuert werden. Jedenfalls besteht derzeit keine Veranlassung, die motivierende Kraft von Subventionen als ein funktionales Äquivalent für die rechtsnormative Konkretisierung einer „dienenden Funktion“ des Rundfunks im deutschen Kommunikationsverfassungsrecht anzusehen. 
In grundrechtsdogmatischer Hinsicht führt diese Rundfunkkonzeption dazu, dass mit zweierlei Rundfunk, zweierlei Rundfunkgewährleistungen, zweierlei funktionalen Ausrichtungen (sc. Meinungsbildung vs. Ratings) des Rundfunks zu rechnen ist (Stock, 2001, S. 124 f.). Kommerzieller Rundfunk ist nun allerdings in funktionaler Hinsicht beliebigen Mediendiensten bis zur Ununterscheidbarkeit nahe gerückt. Damit sind zum einen kompetenzrechtliche Konsequenzen zu überlegen: Bundeszuständigkeit für Telekommunikation und Wirtschaft (Art. 73 Ziff. 7, Art. 74 Ziff. 11 GG; Vesting, 2001, 295)? Zum anderen sind in dem Umfang, in dem die Merkmale der „Darbietung“ und des meinungsbildenden Charakters immer weniger ein zerstückeltes, individualisiertes und „banalisiertes“ (Bullinger, 2001, S. 203; ausführlicher ders., 1980, S. 53 ff.) kommerzielles Angebot kennzeichnen sollen, der Charakter dieses Angebots als „Rundfunkprogramm" und die an diesen Charakter gebundenen Privilegien - vor allem im Hinblick auf Übertragungsmöglichkeiten - zu überprüfen. Im Übrigen wäre wohl auch die Möglichkeit in Rechnung zu stellen, dass im dauerhaften Konsum der Angebote solcher als Rundfunkprogramm getarnter Mediendienstleistungen die Kompetenz zur selbstbestimmten Wahrnehmung, Unterscheidung und Beurteilung medialer Angebote auf Dauer beschädigt werden könnte. Die Rezipienten könnten schließlich infolge immer weiter verminderter „Medienkompetenz“ (hierzu Lauffer/Volkmer, 1995; Fromm u. a., 2000) durch ein anspruchsvolles Qualitätsprogramm schon gar nicht mehr erreicht, jedenfalls von vornherein überfordert werden. Die Meinungsbildungsfreiheit aller Rezipienten ist grundrechtlich aber nicht nur in ihren Bestands-, sondern auch in ihren Entstehensbedingungen geschützt (Rossen, 1988, S. 112 ff.). Die verfassungsrechtliche Legitimation getarnter Mediendienstleistungen, deren Konsum die Entwicklung von Medienkompetenz ausschließt oder hindert, erscheint deshalb zweifelhaft.

Nur der öffentlich-rechtliche Rundfunk ist in dieser Konzeption noch der Gewährleistung freier, umfassender und chancengleicher Meinungsbildung in öffentlichen und privaten Bezügen „dienend“ zugeordnet. In der dogmatischen Konstruktion eines Gewährleistungsgefüges, das von der Basisgewährleistung des Kommunikationsverfassungsrechts zusammengehalten wird (Rossen, 1988, S. 167 ff., 317 ff.), zeigen sich Erosionserscheinungen. Die Einheit dieses Gewährleistungsgefüges zerbricht, wenn für einen bestimmten Rundfunktyp kein Ort mehr in diesem Gefüge, keine konstitutive Beziehung zur Meinungsbildungsfreiheit mehr rekonstruiert werden kann. Letztlich bleibt dabei auch der öffentlich-rechtliche Rundfunk nicht unberührt. Grundrechtsdogmatisch ist nicht mehr erkennbar, in welcher spezifisch verfassungsrechtlichen Grundlage die „besondere Pflichtenstellung“ wurzeln könnte, die dem öffentlich-rechtlichen Rundfunk in seiner Kulturnische hier noch zugeschrieben wird. Wenn die Meinungsbildungsfreiheit für Ausgestaltung und Praxis des kommerziellen Rundfunks kein normativer Bezugspunkt mehr sein soll, warum dann für den öffentlich-rechtlichen Rundfunk? Die hier vorgestellte Konzeption beruht auf einer Meinungsbildungsfreiheit, die in ihrer rechtlich-maßstäblichen Bedeutung aus Gründen halbiert ist, die ihrerseits grundrechtsdogmatisch nicht rekonstruiert werden können (dazu Grimm, 2001, S. 29). Die Verfassung, die nach Wortlaut und Telos des Art. 5 Abs. 1 S. 2 GG keine typologisch-funktionale Unterscheidung innerhalb des Rundfunkbegriffs vornimmt oder zulässt, ist bereits mit den unterschiedlichen Anforderungsniveaus für öffentlich-rechtlichen und kommerziellen Rundfunk (BVerfGE 73, 118 [157]) aufs Äußerste strapaziert worden (Rossen, 1988, S. 375 ff.). Sie gerät als Grundlage spezifisch rechtlicher Unterscheidungen jetzt ganz außer Sicht. An die Stelle verfassungsrechtlicher und grundrechtsdogmatischer Argumente tritt das Anerkenntnis der Übermacht ökonomischer Mechanismen. 


\section{c) Ökonomische Kolonisierung, verfassungsrechtliche Delegitimation}

Verfassungsrecht, auch und gerade Kommunikationsverfassungsrecht, hat an der Spitze (oder Grundlage) der Rechtsordnung die Funktion, die Möglichkeit - wenn auch nicht den Erfolg - des rechtlichen Arguments gegen die Wucht anderer funktionssystemspezifischer Imperative offen zu halten und abzusichern (Rossen-Stadtfeld, 2000, S. 182 ff., 184 ff.). Es handelt sich um eine der verschiedenen Vorkehrungen moderner Gesellschaften, das historisch erreichte Maß an funktionaler Differenzierung nach Möglichkeit aufrecht erhalten zu können. Mit diesem Maß verknüpft ist aber auch das Maß an Vielfalt und Heterogenität der Perspektiven, Optionen, Maßstäbe und „Weltsichten“, das eine Gesellschaft zu einem bestimmten Zeitpunkt kennzeichnet. Wo der Geltungsbereich des verfassungsrechtlichen Arguments in der beschriebenen Weise zugunsten ökonomischer Gesichtspunkte verkürzt wird, findet Entdifferenzierung statt. Die betroffene Gesellschaft wird dann dimensional öder und verarmt. Eine Gesellschaft, die sich zunehmend nur noch nach Maßgabe und in den Grenzen der systemischen Imperative ihrer Ökonomie marktförmig integriert, ist weit davon entfernt, einer Vielfalt von Beobachtungsperspektiven, Orientierungsmodellen und Entscheidungsalternativen wirklich Raum geben zu können (scharfsichtig hierzu Ross, 1997, S. 99). Sie verliert aber auch an Potenzial, die problematischen, dysfunktionalen und womöglich zerstörerischen Nebenfolgen eines ungebremsten ökonomischen Diskurses in ihren Medien kenntlich zu machen und dann vielleicht Wege zu finden, diese Nebenfolgen auszubalancieren. Niemals wird etwa die unter ökonomischen Gesichtspunkten (Werberatings, Konsumanreize, endloser/unbegrenzter Bedarf bei stets nur kurzfristiger Befriedigung etc.) jederzeit vorzugswürdig erscheinende "Spaßgesellschaft" den unerfüllten utopischen Überschuss ihres bildungsbürgerlichen Herkommens aufnehmen und umsetzen können. Ebenso wenig kann sie neue Probleme auch nur in Umrissen fassen, mit denen es die Gegenwart immer drängender zu tun bekommt (etwa: Entwicklungsungleichzeitigkeiten innerhalb „multikultureller“ Gesellschaften, Persönlichkeitsentwicklung ohne vorgegebene Rollenschemata und außerhalb einer herkömmlichen Erwerbsbiografie, Jungsein und Altwerden ohne sozialen Ort, aber auch jüngere Fragen wie die nach einer gentechnisch erweiterten Perfektibilität des Menschen und seiner Umwelt).

Die Konzeption einer Übersteigerung des dualen Systems wirft noch weitere Fragen auf. Wenn kommerzielle und öffentlich-rechtlich verantwortete Angebote unter verschiedene Pflichtenregime gestellt, wenn die beiden Sektoren des dualen Systems weiter und deutlicher auseinander gezogen werden sollen, dann bedarf es juristisch handhabbarer Kriterien, anhand deren die Trennlinie in Konfliktfällen ausgezeichnet werden kann. Diese Kriterien können sich, da der kommerzielle Rundfunk dereguliert und von (noch bestehenden) Pflichtenbindungen nach Möglichkeit freigestellt werden soll, nur auf den öffentlich-rechtlichen Rundfunk beziehen. Es muss also festgelegt werden, was dieser Rundfunk unter der leitenden Maßgabe „gesteigerter Pflichtenbindung“ zu leisten und worin er sein spezifisches programmliches Profil zu finden hat. Diese Festlegung eines öffentlich-rechtlichen „Funktionsauftrags“ (als Nachfolger der älteren „Grundversorgung“) wiederum kann in dem Ansatz dieser Konzeption nicht dem Rundfunk überlassen bleiben. Sie stellt eine grundrechtswesentliche Aufgabe dar, die der Gesetzgeber selbst bearbeiten muss, indem er den Programmauftrag über das heute schon erreichte Maß hinaus weiter konkretisiert. Auch wenn das in dieser Deutlichkeit nicht ausgesprochen wird: Der öffentlich-rechtliche Rundfunk verlöre letztlich die ihm derzeit noch unbestritten verfassungsrechtlich garantierte Programmautonomie (Eifert, 2002, S. 89 ff.; Hoffmann-Riem, 2000, S. 188 f.). 
Es ist also eine beträchtliche Verschiebung der grundrechtsnormativen Gewichte, der hier das Wort geredet wird. Sie wiegt umso schwerer, als keineswegs ausgemacht erscheint, dass quantitative oder inhaltsbezogen-qualitative Festlegungen über den (niedrigen) Genauigkeitsgrad des derzeitigen Programmrechts hinaus tatsächlich steuerungswirksam werden, in der Begrifflichkeit der hier behandelten Konzeption also eine "gestärkte Pflichtenbindung “ bewirken könnten. Die derzeit verfügbaren sozialwissenschaftlichen Daten sprechen dagegen (Eifert, 2002, S. 44 ff. m. w. N.). Ein öffentlichrechtlicher Rundfunk, der auf ein möglichst hohes Qualitätsniveau in seinen Programmen verpflichtet werden soll, bedarf verbesserter Einrichtungen der Selbststeuerung und Selbstkontrolle, eines moderneren Qualitätsmanagements, vielleicht auch eines intensivierten Zusammenspiels interner und externer Programmbeobachtung (Vorschläge bei Eifert, 2002, S. 113 ff. m. w. N.). Weniger dringend bedarf er eines weiter konkretisierten, ausdifferenzierten und so zur Bereichsabgrenzung tauglichen Programmrechts.

\section{Die Aufhebung: Markt statt Recht, „Kultur“ statt Meinungsbildung}

Auch ein zweiter Vorschlag zur Zukunft des dualen Rundfunksystems knüpft bei dem beschleunigten Wandel an, der sich im Realbereich der normativen Gewährleistungen des Art. 5 Abs. 1 GG derzeit vollzieht (Vesting, 2001a). Dargestellt wird der Wandel in dieser Konzeption unter dem Leitbegriff einer „Logik der Vernetzung“. Gemeint ist damit, dass die medientechnische Entwicklung (insbesondere: Internet) und das Interesse an der Maximierung der Einschaltquoten, der Aufmerksamkeit und damit der Werbeerlöse ein Verhältnis wechselseitiger Beförderung eingingen. In ihm löse sich die Unterscheidung zwischen Rundfunk, Telekommunikations- und Mediendiensten immer stärker auf, verliere die Veranstalterfunktion in langen und unübersichtlichen Verwertungsketten an Bedeutung, erweise sich die Prominenz von Personen und Themen als neue „Währung“ des Mediensystems und sei eine Steuerung des dualen Rundfunksystems durch „positives“, an der verfassungsrechtlichen Vorgabe der Meinungsbildungsfreiheit orientiertes Rundfunkrecht nicht mehr möglich (a. a. O., S. 287 ff.). Die Schilderung der Wandlungsprozesse deckt sich mit der Darstellung, von der die Zuspitzungs-Konzeption ausgeht (neue Techniken, Entdifferenzierung, Verwischung herkömmlicher Unterscheidungen, Ökonomisierungsdruck, Konvergenztendenzen). Die Konsequenzen, die aus dieser Schilderung des Wandels gezogen werden, sind gleichfalls problematisch.

\section{a) Keine Regulierung gegen die „Logik der Vernetzung“}

Eine Grundthese lautet, dass das moderne duale Rundfunksystem sich weder politisch verantworten noch rechtlich regulieren lasse (a. a. O., S. 294). Der Zugang zu einem neuen Rundfunkmodell sei in einer Perspektive der „Erhaltung gesellschaftlicher und vor allem wirtschaftlicher Innovationsfähigkeit“ zu suchen, nicht aber mehr über eine „staatszentrierte Vorstellung von ,Meinungsbildung‘ oder über Begriffe wie ,Zugangsgerechtigkeit' oder, Chancengleichheit"“ (a. a. O., S. 296). Der These fehlt die überzeugende Begründung. Im theoretischen Entwurf mag „die Logik der Vernetzung durch eine Steigerung der Optionenräume die Diskriminierungskapazität von Grenzbegriffen" unterlaufen (a. a. O., S. 289). Im Zielbereich der Konzentrations- und Vermachtungsschübe, die im Zusammenbruch der New Economy derzeit zu beobachten sind, aber auch etwa in dem unter Quotendruck und Werbeflaute zunehmend „einfältiger“ 
gestalteten kommerziellen Programmangebot, wird es freilich noch eine Weile dauern, bis sich von dort her ausgeweitete „Optionenräume“ abzeichnen könnten. Immerhin bleibt auch dann noch die Erkenntnis, dass dieselbe digitale Übertragungstechnik schon jetzt ganz unterschiedliche Programm- und Dienstleistungsangebote zu befördern geeignet ist. Bei dieser Technik anknüpfende Abgrenzungen geraten also in die Gefahr, kein klares Substrat mehr zu finden. Regulierung, die auf trennscharfe Rechtsbegriffe angewiesen ist, stößt hier in der Tat auf Schwierigkeiten. Dass diese aber sogleich zur Unmöglichkeit der Regulierung führen sollen, ist damit noch nicht zureichend nachgewiesen. Insbesondere in dem wichtigen Teilbereich der rechtlichen Erfassung, Einordnung und Steuerung von Online-Leistungen des Rundfunks sind ausführliche und differenzierte Vorschläge zu Regulationsansätzen vorgelegt worden (Hoffmann-Riem, 2000, S. 229 ff., 233 ff.). Sie zeigen, dass Regulationsbemühungen jedenfalls nicht schon von vornherein als aussichtslos angesehen werden müssen. Wiederum unbestreitbar sind ferner eine immer wieder versagende Praxis der internen („gesellschaftlichen“) Rundfunkkontrolle und der externen Medienaufsicht sowie schließlich die Feststellung einer - freilich in Ausmaß und Dimensionen sehr umstrittenen und erläuterungsbedürftigen - „Konvergenz nach unten“ im Gesamtprogrammangebot des dualen Rundfunksystems. Solche Mängelbefunde können auch (niemals allein) in zu wenig, in zu viel, in schlechter oder - der Regelfall - in unzureichend durchgesetzter rechtlicher Regulation (Zulassung, Programmaufsicht, Konzentrationskontrolle) ihre Ursachen finden. Die Grundthese einer unmöglich werdenden Rundfunkregulierung durch Recht lässt sich durch sie also kaum schon ausreichend stützen.

Allerdings wird auch auf eine „Logik der Vernetzung“ (a. a. O., S. 287; Begriff schon bei Ladeur, 1999, S. 68 ff.) verwiesen, die Regulierungskonzepte nach Art des Rundfunkstaatsvertrages ins Unwirkliche und Unvollziehbare treibe. Dieser Verweis impliziert die Vorstellung eines unentrinnbaren Geschicks („Logik“), dem die Gesellschaft preisgegeben sei. Die Beiläufigkeit, mit der Meinungsbildungsfreiheit, Chancengleichheit und Zugangsgerechtigkeit als normativ und konzeptionell leitende Gesichtspunkte beiseite geschoben werden und stattdessen „Innovationsfähigkeit“ in den Mittelpunkt gerückt wird, könnte in einer solchen Hintergrundvorstellung ihre Erklärung finden. Wo aber heute die Steigerung von „Innovationsfähigkeit“ gefordert oder ähnliche Schreckworte (etwa: „Flexibilität“, „Dynamik“, „Herausforderung“) gebraucht werden, heißt es aufmerken. Meist soll den so Angesprochenen angezeigt werden, dass härtere Zeiten bevorstehen, und zwar vor allem ihnen. Dementsprechend ergeht auch in dieser Rundfunkkonzeption eine ernste Ermahnung an den öffentlich-rechtlichen Rundfunk, sich „im Wettbewerb der Aufmerksamkeit“ nicht auf „Strategien der Marktverstopfung“ einzulassen (a. a. O., S. 293, 304 f.; ebenso Ladeur, 2000). Ob das im Niedergang des dualen Systems wirklich ein aktuelles Zentralproblem darstellt, ob demgegenüber nicht eher darauf $\mathrm{zu}$ achten wäre, eine ebenso bedrohte wie für moderne Gesellschaften unverzichtbare Public Service-Tradition gegen den immer mächtiger werdenden Kommerzialisierungsdruck zu verteidigen, soll hier dahingestellt bleiben. Die Erwägung, dass gesellschaftliche Entwicklung, wenn schon nicht gesteuert, so doch nach Maßgabe einigermaßen freier, aufgeklärter und vernünftiger Entscheidungen immerhin beeinflusst werden könnte, liegt jedenfalls weit jenseits der Perspektive, die mit dieser Konzeption zur Entwicklung des dualen Rundfunksystems eröffnet wird. Dieser Rundfunkkonzeption geht es in erster Linie um die Anpassung der Kommunikationsstrukturen moderner Gesellschaften an das, was auch so schon unwiderstehlich genug erscheint: die funktionalen Imperative von Wirtschaft und Technik. Anderes als diese Anpassung befördere nur die „Flucht in abstrakte Glaubensbekenntnisse“ (a. a. O., S. 295). 


\section{b) Ein problematischer Kulturbegriff}

Damit gerät diese Konzeption in die Gefahr, sich selbst den Boden unter den Füßen wegzuziehen. Sie hat nämlich durchaus ein Regulierungsanliegen. Eine zweite Grundthese dieser Konzeption geht zunächst dahin, dass die Abgrenzung zwischen öffentlichrechtlichem und kommerziellem Rundfunk ohnehin längst fiktiv geworden sei, so dass auf sie verzichtet werden solle (a. a. O., S. 293). Sodann sei für das derart wieder vereinheitlichte Rundfunksystem ein „neues rundfunkrechtliches Ordnungsmodell“ zu entwerfen. Dieses dürfe keinesfalls mehr auf die Gewährleistung freier, umfassender und chancengleicher Meinungsbildung in öffentlichen und privaten Bezügen ausgerichtet sein und darin alteuropäisch-bildungsbürgerlich verengt werden. Stattdessen sei die leitende Maßgabe des neuen Ordnungsmodells künftig allein in der „Sicherung kultureller Vielfalt" zu sehen (a. a. O., S. 296). Hier also soll durchaus noch reguliert werden, wenn auch erst nach einer „Verschiebung des verfassungsrechtlichen Ansatzpunktes von Politik auf Kultur bzw. auf die Erhaltung kultureller und wirtschaftlicher Innovationsfähigkeit“" (a. a. O., S. 298).

Wenn die bisherige Grundnorm des sich aus Art. 5 Abs. 1, 2 GG ergebenden Kommunikationsverfassungsrechts, die Gewährleistung der Meinungsbildungsfreiheit, derart entschieden durch einen Leitwert „Kultur“ ersetzt werden kann, wird man hierfür eine spezifisch verfassungsrechtliche Begründung erwarten dürfen. Die hier vorgestellte Rundfunkkonzeption setzt eine solche Begründung schon voraus. Für sie ist ausgemacht, dass auf „rechts- und verfassungstheoretischer Ebene ... ein solches Konzept unterstellen [kann], dass es ein öffentliches Interesse an der Sicherung kultureller Vielfalt gibt“ (a. a. O., S. 296). Als deskriptiv-empirische ist diese Feststellung keineswegs selbstverständlich. Seit jeher und nicht nur in Deutschland hat „kulturelle Vielfalt" als Politikziel ohnehin keinen guten Stand. Derzeit aber kann in Deutschland von einem öffentlichen Interesse an ihr etwa im Hinblick auf die öffentliche Finanznot, auf vordringliche Arbeitsmarktprobleme und weitere damit verbundene Verschiebungen im gesellschaftlichen Präferenzhaushalt, auf innenministerielle Assimilationsforderungen oder auch etwa auf das Programmangebot des kommerziellen Rundfunks keine Rede sein. Wenn überhaupt in der parteipolitischen oder auch medienrechtlichen Diskussion die Forderung nach kultureller Vielfalt erhoben wird, dann scheint dies in erster Linie der Ungreifbarkeit und Plastizität beider Wörter geschuldet. Ihr operationalisierbarverpflichtender Gehalt geht nicht über die Substanz hinaus, die etwa Versprechen einer „wirksamen Bekämpfung der Massenarbeitslosigkeit“, der „Sicherung des Weltfriedens“ oder einer „nachhaltigen Verbesserung der Umweltqualität“ aufweisen. Ein öffentliches Interesse an kultureller Vielfalt bleibt also zumindest äußerst zweifelhaft.

Die Empfehlung, das Rundfunkverfassungsrecht auf „Kultur“ umzustellen, wird noch auf ein zweites Argument gestützt. Dabei werden „biologische Diversität“ als „Voraussetzung für die Evolution lebender Systeme“ einerseits und „kulturelle Vielfalt“ als „Voraussetzung für die Sicherung der laufenden Selbsterneuerung einer (post-)modernen Gesellschaft" andererseits parallelisiert (a. a. O., S. 296). Biologismen indizieren Begründungsprobleme, als Argumente sind sie nutzlos. Die hier diskutierte Rundfunkkonzeption schiebt aber ein drittes Argument nach. Das öffentliche Interesse an der Erhaltung kultureller Vielfalt lasse sich ,auch deshalb schwerlich in Frage stellen, weil die Erhaltung der Innovationsfähigkeit der Kultur für die Innovationsfähigkeit der Wirtschaft mehr und mehr von ausschlaggebender Bedeutung ist" (ebd.). Kultur in dienender Funktion der Ökonomie zugeordnet - das könnte praktisch funktionieren, doch dürfte diese neue Art funktionaler Grundrechtsinterpretation mit Wortlaut und Sinn 
des Art. 5 Abs. 1 S. 2 GG kaum mehr in Deckung zu bringen sein. Die Umstellung des Rundfunkverfassungsrechts auf einen Leitwert „kulturelle Vielfalt“ wird also mit einer zweifelhaften Unterstellung, mit einem Biologismus und schließlich ökonomisch begründet. Das Bundesverfassungsgericht entwirft demgegenüber von der Meinungsbildungsfreiheit aus einen Zusammenhang konstruktiv-dogmatisch aufeinander bezogener Teilgewährleistungen des Art. 5 Abs. 1 GG, in den dann schließlich auch eine „kulturelle Verantwortung" des Rundfunks (BVerfGE 90, 60 [90]; s. schon BVerfGE 73, 118 [158]; 74, 297 [324]) eingebaut werden kann, und es sucht diesen Zusammenhang immer wieder in den Realbereichen der ihn tragenden Normen abzusichern. Das ist besser nachzuvollziehen und wohl auch verfassungsrechtlich anschlussfähiger.

Der „Kultur“ kommt in dieser Rundfunkkonzeption eine entwicklungsstrategisch maßgebliche Bedeutung zu. Was aber könnte dieser Begriff hier meinen? Kultur sei „eine Art zerstreutes und flexibles Gedächtnis“, ein „Filter des Erinnerns und Vergessens“, durch den man „je nach Lage und Situation, Vergangenheit in Anspruch nehmen und den Variationsrahmen der Zukunft bestimmen kann“; Kultur sei „dispers und kontingent“ geworden, „von Situationen und Ereignissen abhängig“, auch unterliege sie, unter Beteiligung der Massenmedien, der „dauernden Selbstveränderung“ (Vesting, 2001a, S. 297). Damit wird der in der Tat einzige einigermaßen scharf geschnittene Kulturbegriff aufgenommen, der zurzeit in den Sozialwissenschaften zur Verfügung steht. Bei Lubmann (etwa 1997, S. 409 ff., 587 f.) und Baecker (2000) bezeichnet Kultur den Gesichtspunkt, unter dem einer interessierten Beobachtung alles mit allem vergleichbar wird. Es ist eine wichtige, für moderne pluralistische Gesellschaften wahrscheinlich überlebenswichtige Leistung, die so erbracht werden kann. Es kann alles Fremde und Überraschende zunächst einmal - „wie interessant!“ - in die Wahrnehmung eingebaut werden. Weiteres wird sich finden, nach Maßgabe der Kriterien nämlich, die das Interesse jeweils lenken. Das muss dann nicht immer Erinnern bedeuten. Interesse kann auch abgezogen werden, dann fallen ehedem als kulturell bedeutsam erachtete Gehalte dem Vergessen anheim, auch dies ist eine notwendige Leistung für Gesellschaft und Individuen.

Ein Mediensystem allerdings, dessen oberster verfassungsrechtlicher Ansatzpunkt eine so verstandene Kultur sein soll, gerät in schwerstes Wasser. Dieser Ansatzpunkt ermöglicht schon per definitionem keinen Gegenhalt mehr, wenn etwa quotengestützte Entdifferenzierungs- und Simplifikationszumutungen der „Ökonomie der Aufmerksamkeit“ abgewehrt werden müssten. Zwar legt die hier vorgestellte Konzeption immer wieder einen inneren Zusammenhang zwischen „Kultur“ und „Vielfalt“ nahe (etwa a. a. O., S. 296). Worin dieser Zusammenhang bestehen könnte, wird aber nicht näher erläutert. Der als Referenz benannte Kulturbegriff jedenfalls beinhaltet einen solchen Zusammenhang keineswegs. Ganz im Gegenteil: „Kultur verhindert ... die Überlegung, was man anstelle des Gewohnten anders machen könnte" (Luhmann, 1997, S. 588) Kultur wird hier also als Variationsbremse begriffen, anders könnte sie ja auch kaum zur Praxisform der Unterscheidung zwischen Erinnern und Vergessen werden. Diese „Kultur" auf der einen und „Vielfalt" auf der anderen Seite haben strukturell nichts miteinander zu tun. Kultur ist danach vielmehr das, was nach dritten Maßstäben (im Rundfunk etwa: unterstellter Massengeschmack, Simplizität, Rasanz, Sexappeal, Brutalität, „Starpower“ etc., s. Vesting, 2001a, S. 291 f., 299 f.) als „interessant“ eingestuft und zur Rezeption feilgeboten wird. Was nach diesen Maßstäben als uninteressant gilt, ist für die betreffende Wahrnehmung nicht mehr Kultur. Es kann dann zwar nach anderen Maßstäben und in anderer Wahrnehmung noch als ,interessant“ eingestuft werden. Für die massenmedial gestützte gesellschaftliche Kommunikation bleibt es aber uninteressant 
und damit: nicht existent. Nur folgerichtig ist deshalb der Vorschlag, auf die Vorgabe regulativer Ziele zu verzichten und „sich“ (gemeint ist wohl das Rundfunkrecht) auf „den zeitlichen Fluss der sich von Moment zu Moment vollziehenden ... Selbstreproduktion des Rundfunks und der Medienwirtschaft einzulassen“ (a. a. O., S. 298). Erneut also wendet sich der Blick der Ökonomie zu. Das ist konzeptionell stimmig. Dort in der Tat werden jene dritten Maßstäbe vor allem festgelegt, anhand derer die kulturelle Relevanz von Medienangeboten bestimmt wird - und die, so die konzeptionelle Idee, dann von den rundfunkrechtlichen Maßgaben normativ abgestützt werden müssen.

\section{c) Qualitätsmanagement obne Qualitätsbegriff}

Überraschen muss dann freilich, dass das hier empfohlene neue Rundfunkrecht doch noch veranlassen und fördern soll, was ausdrücklich als „Qualitätsmanagement“ bezeichnet wird (a. a. O., S. 302 f., 304). Wie immer selbstregulativ dieses Management nun auch ausfallen soll, wie immer „kooperativ“ seine Kontrolle zu sein hat, wie immer die Schwellenwerte zur Erzwingung dieses Managements gebildet werden könnten (Andeutungen dazu a. a. O., S. 301 f.) - Qualitätsmanagement benötigt, soll der Begriff nicht ganz inhaltsleer sein, einen Begriff von Qualität. Dieser Begriff muss Maßstäben aufruhen, die eine Unterscheidung zwischen qualitativ Wertvollem und qualitativ Wertlosem ermöglichen. Eben diese Maßstäbe werden einer Medienregulierung aber aus der Hand genommen, der als zentraler Leitwert nur noch „Kultur“ im skizzierten Verständnis vorgegeben ist. Diese Medienregulierung kann gar nicht anders verfahren, als extern bestimmte Maßstäbe zu übernehmen. Wie unter solchen Bedingungen ein programmbezogenes Qualitätsmanagement auch nur entworfen, geschweige denn regulativ durchgesetzt werden könnte, ist nicht zu erkennen.

\section{Meinungsbildungsfreiheit und Public Service}

Beide vorstehend nachgezeichneten Konzeptionen zur weiteren Entwicklung des dualen Systems nehmen bei der Beobachtung eines Wandels der technischen, wirtschaftlichen, soziokulturellen und politischen Rahmenbedingungen des Rundfunks ihren Ausgang. Sie gelangen von dort aus einmal zu einer zuspitzenden Übersteigerung, im anderen Fall zu einer Aufhebung der systemprägenden Dualität. In beiden Fällen, auch in der Variante der Übersteigerung, gibt es dasjenige duale Rundfunksystem, dessen Grundlinien in der Rechtsprechung des Bundesverfassungsgerichts vorgezeichnet worden sind, am Ende nicht mehr. In beiden Fällen sind es ökonomische Interessen und Funktionsimperative, von denen die weitere Entwicklung des Rundfunks maßgeblich bestimmt werden soll. In beiden Fällen schließlich fehlt es den vorgestellten Rundfunkmodellen an einer verfassungsrechtlich tragfähigen Begründung. Im Übersteigerungs-Modell zerbricht mit der Herauslösung des kommerziellen Rundfunks aus den Bindungen, die in der Meinungsbildungsfreiheit ihren Ursprung haben, der Gewährleistungszusammenhang des Art. 5 Abs. 1 GG. Mit diesem entfällt aber nicht zuletzt auch die verfassungsrechtliche Begründung einer gesteigerten Pflichtenbindung des öffentlich-rechtlichen Rundfunks. Der Vorschlag einer vereinheitlichenden Aufhebung des dualen Systems wiederum sucht sich schon von vornherein ausdrücklich und entschieden von allen bislang gültigen kommunikationsverfassungsrechtlichen Vorgaben zu trennen. 


\section{a) Auch meinungsbildende Unterbaltung ...}

Die Konzeption des Bundesverfassungsgerichts erscheint demgegenüber verfassungsrechtlich besser begründet, vielfaltsförderlicher, deutlich realitätszugewandter und vor allem wesentlich entwicklungsfähiger. Meinungsbildung findet danach keineswegs nur in den Bereichen Information und Bildung statt. Sie reicht in "gesellschaftlich-kulturelle Tiefendimensionen" (Stock, 2001, S. 121) hinein. Deshalb kann ihr auch das Unterhaltungsangebot des Rundfunks Grundlage und Substrat werden. Die Gewährleistung der Meinungsbildungsfreiheit ist nicht auf Nation oder Staat bezogen. Sie hat Verfassungsrang erlangt, weil ihr im Hinblick auf die Sicherung gesellschaftlicher Öffentlichkeit und die laufende Persönlichkeitsentwicklung in sozialen Beziehungen Bedeutung zukommt. Schließlich vollzieht sich verfassungsrechtlich geschützte Meinungsbildung keineswegs nur in öffentlichen Räumen, sondern auch in individuell-privaten Innenwelten. Sie wird dabei zum Konstituens nicht nur der Demokratie, sondern gerade auch unverfügbarer personaler Subjektivität. Das alles wird in den konzeptionell maßgeblichen Entscheidungen des Gerichts seit langem als selbstverständlich mitgeführt (s. etwa BVerfGE 35, 202 [222 f.]; 57, 295 [319]; 73, 118 [152]; st. Rspr.).

Auch der Verdacht, dass die in der Meinungsbildungsfreiheit zentrierte Konzeption des Bundesverfassungsgerichts kognitiv- „politisch“ und etatistisch verengt sei (Vesting, 2001b, S. 230 f.), ist deshalb unbegründet. Die Dimensionen, in denen Meinungsbildung verfassungsrechtlichen Schutz genießt, sind mit dem Begriff der Meinungsbildungsfreiheit nicht festgelegt. Auch sie sind dem offenen, niemals abgeschlossenen Prozess überantwortet, den dieser Begriff bezeichnet (Eifert, 2002, S. 23 f.). Es lässt sich ein Konzept verfassungsrechtlicher Vorgaben für die Rundfunkordnung entwerfen, das wichtige Funktionen der Massenmedien für den politischen Prozess anerkennt, ohne deshalb ebenso wichtige Funktionen derselben Massenmedien für die Aufrechterhaltung zivilgesellschaftlicher, staatsvergessener Pluralität und Selbstbestimmungsfähigkeit sowie für die Entwicklung und laufende Selbstvergewisserung bürgerlicher Subjektivität leugnen zu müssen (Rossen, 1988, S. 97 ff., 109 ff., 112 ff., 317 ff.). Die Rechtsprechung des Bundesverfassungsgerichts kann in einem solchen Konzept abgestützt werden. In ihr wird vielfach auf die Bedeutung der Massenmedien für die Orientierung der Einzelnen in ihren gesellschaftlichen Beziehungen (die den demos politikos, wenn man Luhmann folgen will, einschließen mögen, also über ihn hinausreichen) und für die Entwicklung der individuellen Persönlichkeit hingewiesen (s. o.).

\section{b) ... als Gegenstand der Medium-und-Faktor-Funktion des Rundfunks}

Der Bereich, in dem sich Meinungsbildung ereignet und in dem deshalb der Rundfunk eine „dienende Funktion“ innehat, wird damit allerdings in der Tat sehr weit ausgezogen. Dies ist die Konsequenz einer grundrechtsdogmatischen Konzeption, die das Ziel freier, umfassender und chancengleicher Meinungsbildung in öffentlichen und in privaten Bezügen in seiner ganzen Vielschichtigkeit ernst zu nehmen sucht. Eines der großen Rätsel einer einfachrechtlich konkretisierten Medienverfassung, die eine lebendige, reiche und vielfältige gesellschaftliche Kommunikation sicherstellen will, besteht gerade darin, wie die unverfügbare Subjektivität der Kommunikationsteilnehmer anerkannt und gewährleistet, zugleich aber auch zur Beförderung des „gemeinen Wesens“ einer Gesellschaft befähigt und motiviert werden könnte, die zutiefst differenziert, pluralisiert und individualisiert ist und das auch bleiben soll. Die FRAG-Entscheidung (BVerfGE 57, 295 [319 ff.]) ist deshalb eine der besseren Entscheidungen des Bundesverfassungs- 
gerichts, weil sie die Spannung aufnimmt, die von jenem Rätsel ausgeht. Diese Entscheidung, fortgeführt vor allem in dem NRW-Urteil (BVerfGE 83, 238 [295 ff.]), lässt vereinfachende Marktrundfunktheoreme ebenso wie jeden paternalistischen Gestus weit hinter sich. Sie legt zur Lösung des Rätsels vielmehr eine Konstruktion nahe, in der eine mediale und faktorielle Funktion des Rundfunks normativ auf die gleiche umfassende Meinungsbildungsfreiheit aller ausgerichtet und zur eigenständigen, professionellen und organisierten Bearbeitung in besonderen Berufsrollen ausdifferenziert ist. Die Wahrnehmung dieser Funktion kann (nicht: muss!) das ihre dazu beitragen, dass selbstbewusste Persönlichkeiten an vielfältigsten Kommunikationen teilhaben können (nicht: müssen!), nicht zuletzt also auch an denjenigen Kommunikationen, in denen unter kulturellen, sozialen, ästhetischen, politischen oder wirtschaftlichen Gesichtspunkten Meinungen zur weiteren Ausgestaltung gesellschaftlicher Kommunikation gebildet werden. Was gelingende Meinungsbildung ausmachen könnte, soll sich (und kann sich auch nur, s. Eifert, 2002, S. 36, 49 ff., 143 ff. ) nach diesem Modell in professionell ermöglichten bzw. vermittelten Prozessen freier und umfassender Meinungsbildung, an denen alle teilhaben können (nicht: müssen!), herausstellen. Die neueren Entwürfe zur Zukunft des dualen Rundfunksystems, die oben diskutiert worden sind, bleiben weit hinter der Komplexität des verfassungsgerichtlichen Modells zurück; sie nähern sich dem Rätsel nicht einmal von ferne, auf das dieses Modell schon eine Antwort vorschlägt.

\section{c) Public Service als Verpflichtung}

Der Markt ist jedenfalls kein Medium, in dem dieser Gefahr durchgreifend begegnet werden könnte. Das wird in rundfunkökonomisch instruierten Diskussionsbeiträgen zur Entwicklung des dualen Systems durchaus gesehen, ebenso wird dort zum Teil auch die Notwendigkeit einer normativen Ausrichtung dieser Entwicklung eingeräumt (zusammenfassend Eifert, 2002, S. 61 ff. m. w. N.). Das Leitmodell, das sich hierbei abzeichnet, nimmt eine ältere Tradition auf, um sie in gewandelte Rahmenbedingungen fortzuführen. Es ist die Tradition des Rundfunks als Public Service (dazu Jarren u. a., 2001a, S. 35 ff. m. w. N.), in der das Bundesverfassungsgericht dem Rundfunk eine dienende Funktion als Medium und Faktor freier öffentlicher und privater Meinungsbildung zugeschrieben hat. In dieser Tradition kann (und muss) das verfassungsgerichtliche Leitmodell weiterentwickelt werden. Vorschläge hierzu liegen, wie bemerkt, bereits vor (Hoffmann-Riem, 2000, Teile V-VII; Jarren/ Donges, 2001; für den öffentlich-rechtlichen Rundfunk Jarren u. a., 2001a, S. 155 ff.; Eifert, 2002, Teil 3; mit Blick auf die Perspektive einer „dienstespezifisch diversifizierten Informationsordnung “ Schulz/Held, 2001, S. 124 ff.). Aus dieser Tradition heraus öffnet sich schließlich auch der einzig gangbare Weg, auf dem von dem deutschen Kommunikationsverfassungsrecht Anregungen für eine tatsächlich zukunftsoffene und zukunftsfähige Medienordnung Europas ausgehen könnten (Stock, 2000, S. 53 ff. u. pass.).

\section{Literatur}

Albers. Marion (1996): Zur Neukonzeption des grundrechtlichen »Datenschutzes«, in: Haratsch, A./Kugelmann, D./Repkewitz, U. (Hrsg.), Herausforderungen an das Recht der Informationsgesellschaft, Stuttgart u. a., S. $113-128$.

Baecker, Dirk (2000): Wozu Kultur?, Berlin.

Bullinger, Martin (1980): Kommunikationsfreiheit im Strukturwandel der Telekommunikation, Baden-Baden. 
Bullinger, Martin (1999): Die Aufgaben des öffentlichen Rundfunks. Wege zu einem Funktionsauftrag, Gütersloh.

Bullinger, Martin (2001): Medien, Pressefreiheit, Rundfunkverfassung, in: Badura, Peter/Dreier, Horst (Hrsg.), Festschrift 50 Jahre Bundesverfassungsgericht, Tübingen, S. 193 - 218.

Bullinger, Martin/Mestmäcker, Ernst-Joachim (1996): Multimediadienste, Baden-Baden.

Determann, Lothar (1999): Kommunikationsfreiheit im Internet, Baden-Baden.

Dörr, Dieter/Janik, Viktor/Zorn, Nicole (2002): Der Zugang zu den Kabelnetzen und die Regelungen des europäischen Rechts, in: Die Landesmedienanstalten (Hrsg.), Der Zugang zum digitalen Kabel, DLM-Schriftenreihe Bd. 22, Berlin.

Eifert, Martin (2002): Konkretisierung des Programmauftrags des öffentlich-rechtlichen Rundfunks, Baden-Baden.

Engel, Christoph (1996): Medienordnungsrecht, Baden-Baden.

Franck, Götz (1998): Ökonomie der Aufmerksamkeit, München.

Fromm, Martin/Haase, Frank/Schlottke, Peter F. (Hrsg.) (2000): Inszenierte Wirklichkeiten - Lernen und Entwicklung in der Medienwelt, Baden-Baden.

Grimm, Dieter (2001): Steuerung medienvermittelter Kommunikation - Verfassungsrechtliche Grundlagen, in: Rossen-Stadtfeld, H./Wieland, J. (Hrsg.), Steuerung medienvermittelter Kommunikation, Baden-Baden, S. $25-33$.

Heinrich, Jürgen (1999): Medienökonomie, Bd. 2: Hörfunk und Fernsehen, Opladen/Wiesbaden.

Hoffmann-Riem, Wolfgang (2000): Regulierung der dualen Rundfunkordnung, Baden-Baden.

Hofstadter, Douglas (1985): Gödel, Escher, Bach, 5. Aufl., Stuttgart.

Holznagel, Bernd (1999): Der spezifische Funktionsauftrag des Zweiten Deutschen Fernsehens (ZDF), Mainz.

Holznagel, Bernd (2002): Konvergenz der Medien - Herausforderungen an das Recht, NJW, S. $2351-2356$.

Jarren, Otfried/Donges, Patrick (2001): Medienregulierung als gesellschaftliche Aufgabe?, in: Rossen-Stadtfeld, H./Wieland, J. (Hrsg.), Steuerung medienvermittelter Kommunikation, BadenBaden, S. $35-50$.

Jarren, Otfried/Donges, Patrick/Künzler, Matthias/Schulz, Wolfgang/Held, Thorsten/Jürgens, Uwe (2001a): Der öffentliche Rundfunk im Netzwerk von Politik, Wirtschaft und Gesellschaft, Baden-Baden.

Kiefer, Marie-Louise (1994): Wettbewerb im dualen Rundfunksystem, Media Perspektiven, S. 430 -438 .

Krönig, Jürgen (2001): Tanz ums goldene Kalb. Globales TV: Die Armen schauen, die Reichen senden, epd medien Nr. 74 v. 19.09.01, S. 3 f.

Kuhlen, Rainer (1995): Informationsmarkt. Chancen und Risiken der Kommerzialisierung von Wissen, Schriften zur Informationswissenschaft 15, Konstanz.

Ladeur, Karl-Heinz (1999): Die Regulierung von Telekommunikation und Medien im Zeitalter ihrer Konvergenz: das Beispiel des Universal Mobile Telecommunications System (UMTS), RTkom 1999, S. $68-75$.

Ladeur, Karl-Heinz (2000): Der „Funktionsauftrag“ des öffentlich-rechtlichen Rundfunks - auf „Integration“ festgelegt oder selbst definiert?, M\&K 2000, S. 93 - 96.

Lauff, Werner (2002): „Einer klingelt an der Tür“. Kabel in Not: ein epd-Interview mit dem Unternehmensberater Werner Lauff, epd medien, Nr. 60 v. 3.8.2002, S. $3 \mathrm{ff}$.

Lauffer, Jürgen/Volkmer, Ingrid (Hrsg.) (1995): Kommunikative Kompetenz in einer sich verändernden Medienwelt, Opladen.

Lerg, Winfried B. (1981): Verdrängen oder ergänzen die Medien einander? Innovation und Wandel in Mediensystemen, Publizistik 26, S. $193-201$.

Luhmann, Niklas (1984): Soziale Systeme, Frankfurt a. M.

Luhmann, Niklas (1997): Die Gesellschaft der Gesellschaft, Bd. 1, Frankfurt a. M.

Metze-Mangold, Verena (2001): Digitale Zeitenwende. Zur politischen Bedeutung zivilisatorischer Regeln, epd medien Nr. 79 v. 6.10.2001, S. 23 - 31.

Michalski, Hans-Jürgen (1997): Die Geburtsstätte einer Zweiten Renaissance? Die „Informations- 
gesellschaft" aus politökonomischer Perspektive, Rundfunk und Fernsehen 45, H. 2, S. 194 214.

Oemichen, Ekkehardt (2002): Offliner 2001 - Internetverweigerer und potenzielle Nutzer, Media Perspektiven, S. $22-33$.

Reimers, Ulrich (1999): Rundfunkpolitik und Technik, in: Schwarzkopf, D. (Hrsg.), Rundfunkpolitik in Deutschland, Bd. 1, München.

Reljič, Dušan (2001): Der Vormarsch der Megamedien und die Kommerzialisierung der Weltöffentlichkeit, in: Brühl, T./Debiel, T./Hamm, B./Hummel, H./Martens, J. (Hrsg.), Die Privatisierung der Weltpolitik, Bonn, S. 58 - 81.

Ridder, Christa-Maria/Engel, Bernhard (2001): Massenkommunikation 2000: Images und Funktionen der Massenmedien im Vergleich, Media Perspektiven, S. $102-125$.

Rötzer, Florian (1999): Megamaschine Wissen. Vision: Überleben im Netz, Frankfurt a. M.

Ross, Jan (1997): Staatsfeindschaft. Anmerkungen zu einem neuen Vulgärliberalismus, Merkur 575, Nr. 2, S. $93-98$.

Rossen, Helge (1988): Freie Meinungsbildung durch den Rundfunk, Baden-Baden.

Rossen-Stadtfeld, Helge (1999): Wissen, Vertrauen, Recht, Kritische Vierteljahresschrift für Gesetzgebung und Rechtswissenschaft, S. $223-238$.

Rossen-Stadtfeld, Helge (2000): Verfassungsgericht und gesellschaftliche Integration, in: Schuppert, G. F./Bumke, Chr. (Hrsg.), Bundesverfassungsgericht und gesellschaftlicher Grundkonsens, Baden-Baden, S. 169 - 195.

Schoch, Friedrich (1998): Öffentlich-rechtliche Rahmenbedingungen einer Informationsordnung, VVDStRL 57, S. 158 - 215.

Schulz, Wolfgang (1998): Rechtsfragen des Datenschutzes bei Online-Kommunikation, http:// www.rrz.uni-hamburg.de/hans-bredow-institut/ws-lehr/aktuelles/lfr-datenschutz.pdf.

Schulz, Wolfgang/Held, Thorsten (2001): Verfassungsrechtliche Rahmenbedingungen einer dienstespezifisch diversifizierten Informationsordnung, in: Kops, M./Schulz, W./Held, Th. (Hrsg.), Von der dualen Rundfunkordnung zur dienstespezifisch diversifizierten Informationsordnung?, Baden-Baden/Hamburg, S. $111-149$.

Starck, Christian (1999): Kommentierung zu Art. 5 Abs. 1, 2, in: v. Mangoldt, H./Klein, F./Starck, Chr. (Hrsg.), Das Bonner Grundgesetz, Bd. 1, 4. Aufl., München.

Steinmüller, Wilhelm (1993): Informationstechnologie und Gesellschaft, Darmstadt.

Stipp, Horst (1996): Eli Noams Cyber TV. Kritische Anmerkungen zu einer Multimedia-Vision, epd/kifu Nr. 19 v. 13.3.1996, S. $4-7$.

Stock, Martin (2000): Medienfreiheit in der EU-Grundrechtscharta: Art. 10 EMRK ergänzen und modernisieren!, Frankfurt a. M.

Stock, Martin (2001): Medienvermittelte Kommunikation: Gestaltungsoptionen und Steuerungsperspektiven, in: Rossen-Stadtfeld, H./Wieland, J. (Hrsg.), Steuerung medienvermittelter Kommunikation, Baden-Baden, S. 117 - 134.

Trute, Hans-Heinrich (1998): Öffentlich-rechtliche Rahmenbedingungen einer Informationsordnung, VVDStRL 57, S. $216-268$.

Vesting, Thomas (1997): Prozedurales Rundfunkrecht, Baden-Baden.

Vesting, Thomas (2001): Das Internet als Herausforderung des „dualen Rundfunksystems“, in: Kops, Manfred/Schulz, Wolfgang/Held, Thorsten (Hrsg.), Von der dualen Rundfunkordnung zur dienstespezifisch diversifizierten Informationsordnung?, Baden-Baden, S. $275 \mathrm{ff}$.

Vesting, Thomas (2001a): Das Rundfunkrecht vor den Herausforderungen der Logik der Vernetzung, Medien \& Kommunikationswissenschaft 49, H. 3, S. 287 - 308.

Vesting, Thomas (2001b): Zur Entwicklung einer „Informationsordnung“, in: Badura, P./Dreier, H. (Hrsg.), Festschrift 50 Jahre Bundesverfassungsgericht, Tübingen, S. $219-240$.

Woldt, Runar (2002): Konturen des digitalen Kabelmarkts, Media Perspektiven, S. 34 - 49. 